\title{
Duvelisib Eliminates CLL B Cells, Impairs CLL- Supporting Cells, and Overcomes Ibrutinib Resistance in Preclinical Models
}

Shih-Shih Chen ( $\sim$ Schen9@northwell.edu )

Karches Center for Oncology Research, the Feinstein Institutes for Medical Research Jacqueline Barrientos

Karches Center for Oncology Research, the Feinstein Institutes for Medical Research

\section{Gerardo Ferrer}

Josep Carreras Leukaemia Research Institute (IJC) https://orcid.org/0000-0002-4084-6815

Priyadarshini Ravichandran

Karches Center for Oncology Research, the Feinstein Institutes for Medical Research

Michael Ibrahim

Karches Center for Oncology Research, the Feinstein Institutes for Medical Research

\section{Yasmine Kieso}

Karches Center for Oncology Research, the Feinstein Institutes for Medical Research

Jeff Kutok

Infinity Pharmaceuticals

Marisa Peluso

Infinity Pharmaceuticals, Inc

\section{Sujata Sharma}

Infinity Pharmaceuticals, Inc

\section{David Weaver}

Institute of Health Sciences, Anhui University

Jonathan Pachter

Verastem Inc.

\section{Kanti Rai}

The Karches Center for Oncology Research. The Feinstein Institutes for Medical Research.

\section{Nicholas Chiorazzi}

Karches Center for Oncology Research, the Feinstein Institutes for Medical Research

\section{Article}

Keywords: chronic lymphocytic leukemia, cancer therapy, Bruton's Tyrosine Kinase (BTKi), phosphoinositide 3-kinase (PI3Ki) 
Posted Date: July 21st, 2021

DOI: https://doi.org/10.21203/rs.3.rs-701669/v1

License: (c) (i) This work is licensed under a Creative Commons Attribution 4.0 International License. Read Full License 


\section{Abstract}

Inhibitors of Bruton's Tyrosine Kinase (BTKi) and phosphoinositide 3-kinase (PI3Ki) have significantly improved therapy of chronic lymphocytic leukemia (CLL). However, the emergence of resistance to BTKi has introduced an unmet therapeutic need. Here we demonstrate in vitro and in vivo the essential roles of PI3K- $\delta$ for CLL B-cell survival and migration and of PI3K- $\gamma$ in T-cell migration and macrophage polarization; and more efficacious inhibition in CLL-cell burden by dual inhibition of PI3K- $\delta, \gamma$. We also report an ibrutinib-resistant CLL case, whose clone exhibited $B T K$ and $P L C \gamma 2$ mutations, responded immediately to single agent duvelisib with a redistribution lymphocytosis followed by a partial clinical remission associated with subsequent modulation of $T$ and myeloid cells. CLL samples from patients progressed on ibrutinib were also responsive to duvelisib in patient-derived xenografts irrespective of BTK mutations. Our data support dual inhibition of PI3K- $\delta, Y$ as a valuable approach for therapeutic interventions, including patients refractory to BTKi.

\section{Introduction}

Chronic lymphocytic leukemia (CLL) results from the clonal expansion and accumulation of $C D 19^{+} C D 5^{+}$ B-lymphocytes ${ }^{1-3}$. Moreover, CLL B cells shape the tumor microenvironment (TME), directly and indirectly, by acting on various TME-cell populations, including T cells, macrophages, mesenchymal stromal cells, follicular dendritic cells, and natural killer cells ${ }^{4-6}$.

Like other functional B cells, CLL cells are activated by signals delivered through multiple receptors, including the $B$ cell receptor for antigen (BCR) and cytokine and chemokine receptors ${ }^{5,7,8}$.

Phosphatidylinositol 3-kinases (PI3K), which play significant roles in malignant B-cell growth ${ }^{9,10}$, signal by four PI3K isoforms $(a, \beta, \delta, \gamma)$, each with distinct functional roles. PI3K- $\delta$ and PI3K- $\gamma$ are specifically expressed in leukocytes ${ }^{11-13}$, with PI3K- $\delta$ to be critical throughout the B-cell lifecycle and essential for survival ${ }^{11,14}$; in contrast, $\mathrm{PI} 3 \mathrm{~K}-\mathrm{y}$ signaling is utilized by non-B cells for chemokine receptor signaling, thereby controlling the migration and localization of $\mathrm{T}$ cells and the polarization of monocytes to immunosuppressive M2 macrophages ${ }^{11,12,15,16}$. These complementary roles of PI3K- $\delta$ and PI3K- $\gamma$ in leukocytes suggest that dual blockade could provide enhanced therapeutic benefits in CLL relative to inhibition of PI3K- $\delta$ alone ${ }^{17}$.

Duvelisib (IPI-145) is a first-in-class, oral, dual PI3K-ס,y inhibitor ${ }^{13}$ that has been approved by FDA for the treatment of patients with refractory or relapsed (R/R) CLL or small lymphocytic lymphoma (SLL) after $\geq$ 2 prior therapies; it is also approved to treat patients with follicular lymphoma after $\geq 2$ prior systemic therapies ${ }^{18}$. Duvelisib potently inhibits PI3K- $\delta$ and PI3K-y in CLL B cells ${ }^{13,19}$, offers a novel approach to treat patients with CLL, potentially enhancing efficacy by targeting both CLL cells and CLL-supporting cells. In vitro, duvelisib sensitizes primary CLL cells to apoptosis and abrogates bone marrow (BM) stromal cell-mediated CLL-cell survival by inhibiting BCR-mediated signaling and chemotaxis ${ }^{17,19}$. 
Of the several approved targeted agents in the CLL treatment armamentarium, the most widely used currently is ibrutinib, a first-in-class Bruton's tyrosine kinase inhibitor (BTKi) approved for first-line and relapsed-disease treatment in CLL and other B-cell malignancies ${ }^{20}$. BTKi is taken continuously until progression of disease or intolerance develops. Resistance to ibrutinib can occur by the development of mutations in the BTK-binding site, e.g., a BTKC481S, and/or in mediators of BCR-signaling downstream of BTK, e.g., PLCG2, which lead to expansion of resistant subclones ${ }^{21-23}$. Mutations in BTK and PLCY2 are found in $\sim 80 \%$ of CLL patients with acquired resistance to ibrutinib ${ }^{24}$. Upon progression in certain patients, therapy is immediately needed as the disease can progress rapidly. Little is known about the role of dual PI3K- $\delta, \gamma$ inhibition as a salvage approach for patients who develop these mutations.

Here we aimed to understand how duvelisib targets TME and treat CLL and BTK-resistant disease as shown in C481S mutant XLA B cell line ${ }^{25}$. We first performed in vitro assays and utilized a patient-derived xenograft (PDX) model of $\mathrm{CLL}^{26-28}$ to explore the mechanisms of action of duvelisib and to characterize the distinct functions of PI3K- $\delta$ and PI3K- $\gamma$ in CLL B cells and CLL-supporting cells. We documented that the two PI3K isoforms played distinct roles in sustaining the survival of CLL cells and found in the PDX model that the inhibitory activity of duvelisib was effective against CLL B cells from patients at various diseases stages and from those whose disease had progressed while receiving ibrutinib, even in the setting of $B T K$ and/or PLCG2 mutations. To substantiate these findings, we also describe the clinical course of a patient who underwent a clinical remission with dual PI3K- $\delta, \gamma$ inhibition after disease progression on ibrutinib.

\section{Results}

\section{Inhibition of PI3K- $\delta$ impairs CLL B-cell proliferation, survival, and migration in vitro and in a PDX model.} We first assessed the distinct contributions of PI3K- $\delta$ and PI3K- $y$ on CLL B-cell survival, proliferation, and

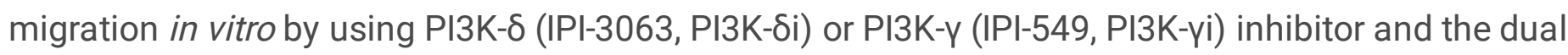
PI3K- $\delta, Y$ inhibitor duvelisib (Table S1).

To evaluate the effects of PI3K-i on CLL B-cell survival in vitro, CLL B cells from four patients was incubated with either 0.1 to $1 \mu \mathrm{M} \mathrm{PI3K}-\delta i$, PI3K-үi, or duvelisib and the absolute numbers of live CLL B cells were quantified after 96 hours. This revealed a significant reduction in viable CLL cells by duvelisib and PI3K-סi with IC50 values at 100nM and $1 \mathrm{uM}$, both doses being achievable and tolerable in patients. However, PI3K-yi did not cause significant changes in CLL cell viability (Fig. 1A).

We next investigated the effects of PI3K- $\delta$ and PI3K-y inhibition on CLL-cell growth. Leukemic B cells were stimulated for 72 hours with soluble (s) CD 40L, interleukin (IL)-2, and IL-10 in the absence or presence of PI3K-סi, PI3K-үi or duvelisib. By measuring the numbers of the phospho-AKT ${ }^{+} \mathrm{Ki} 67^{+}$CLL cells (Fig. 1B), we found that duvelisib $\left(E_{50}=0.46 \mathrm{nM}\right)$ and PI3K- $\delta \mathrm{i}\left(\mathrm{EC}_{50}=0.10 \mathrm{nM}\right)$ more potently blocked CLL B-cell proliferation than PI3K-yi $\left(\mathrm{EC}_{50}=256 \mathrm{nM}\right)$. 
Finally, we assessed the effects of individual or combined PI3K-үi or PI3K-סi on CLL-cell migration in vivo. CLL cells were pre-incubated with 0.1 to $1 \mu \mathrm{M}$ of the PI3K-үi or PI3K-סi or duvelisib for 48 hours, and then injected intravenously (iv) into NSG mice. Twenty-four hours later, the numbers of CLL cells in murine spleens were quantified. Although the PI3K-yi did not significantly affect the migration of CLL B cells into the spleen relative to control, both the PI3K-סi and duvelisib significantly did so in a dose-dependent manner relative to PI3K-yi (Fig. 1C).

Overall, these data indicate the direct effects of PI3K-סi on CLL B-cell survival, proliferation, and migration.

\section{Inhibition of PI3K-Y impairs the ability of T cells and macrophages in the TME to support CLL B-cell} growth and survival. We next investigated the impact of the single versus the dual inhibitory agents on two key components of CLL TME, T cells and myeloid cells.

First, CXCL12-induced T-cell migration was evaluated in vitro ${ }^{17}$. CLL PBMCs treated with various concentrations of DMSO, duvelisib, PI3K-үi (IPI-549), or PI3K-סi (IPI-3063) were allowed to migrate in Transwell plates in response to a 3-hour exposure to CXCL12. Cells were harvested from the lower chamber and the percentage of migrated T cells was quantified. In this assay, PI3K-yi blocked $\mathrm{CD}^{+}$, $\mathrm{CD}^{+}$, or $\mathrm{CD} 8^{+} \mathrm{T}$-cell migration in response to CXCL12 to a greater extent than duvelisib, with PI3K-yi and duvelisib inhibiting at $\mathrm{EC}_{50}$ of $17 \pm 17 \mathrm{nM}$ and $128 \pm 39 \mathrm{nM}$, respectively. PI3Kסi was highly ineffective at inhibiting T-cell migration ( $\mathrm{EC}_{50} 630 \pm 71 \mathrm{nM}$, Fig. $\left.2 \mathrm{~A}\right)$.

Next, migration of CLL-derived T cells was assessed in vivo. CLL-derived T cells were stimulated in vitro by anti-CD3/28 Dynabeads + IL-2 for 7 days in the presence of duvelisib, PI3K-үi or PI3K- $\delta i$, at doses ranging from $0-1 \mu \mathrm{M}$. At the end of culture, duvelisib, PI3K-yi or PI3K-סi treatment didn't change the growth of T-cells (Fig. S1) or surface levels of CXCR4, CXCR5, CCR6 and CCR7 (data not shown). However, when equal number $\left(5 \times 10^{6}\right)$ of T cells from each culture condition were injected iv into NSG mice, T-cell migration to the spleen within 24 hours was blocked by PI3K-үi and duvelisib, but not by PI3K-

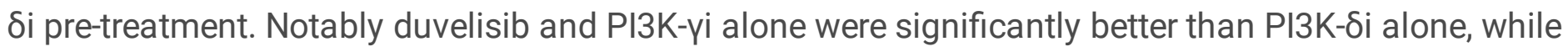
PI3K-yi and duvelisib were not significantly different from each other (Fig. 2B). Thus, PI3K-y function is critical for the migration of CLL-derived T cells.

PI3K-y regulates immune suppression via polarization of myeloid-cells to the M2 phenotype ${ }^{16}$, which is reflected by increased $A r g 1$ (arginase 1) mRNA expression ${ }^{29}$. Thus, we polarized murine BM-derived monocytes (BMDMs) in vitro to M2 macrophages using IL-4 and $\mathrm{M}-\mathrm{CSF}^{30}$ in the absence or presence of duvelisib (Fig. 3A). Duvelisib significantly reduced Arg1 expression at doses equal to or above 10nM, indicating dual inhibition of PI3K-y and PI3K- $\delta$ blocked M2 polarization (Fig. 3B). The same experiment

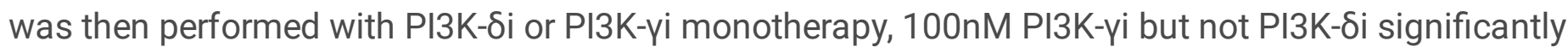
inhibited M2 polarization, while at 1000nM dose, both inhibitors blocked M2 polarization significantly (Fig. S2). 
In CLL, macrophages provide survival signals for the leukemic B cells through PI3K-dependent AKT activation $^{31,32}$. Increasing the M1 to M2 ratio alters the ability of a CLL-cell line to engraft and grow in NSG mice ${ }^{33}$. Therefore, we investigated whether PI3K- $\gamma$ inhibition impeded macrophage-facilitated CLL Bcell survival (Fig. 3C). Co-culturing CLL B cells with M2-polarized murine macrophages for 5 days increased CLL B-cell survival compared with cultures of CLL B cells alone, consistent with M2 macrophages playing a supportive role for CLL B cells (Fig. 3C). Notably, addition of duvelisib to the CLL B cell/M2 macrophage co-culture significantly reduced CLL B-cell survival at doses $>500 \mathrm{nM}$ (Fig. 3C).

In summary, these experiments indicate that inhibition of PI3K-y alone or in combination with PI3K- $\delta$, modulates the numbers and functions of leukemia-supporting T cells and macrophages in the tumor microenvironment.

Dual inhibition of PI3K- $\delta, Y$ reduces the numbers of CLL B cells, CLL-supporting T cells, and myeloid cells in vivo. To assess the impact of duvelisib on transplanted CLL B cells and CLL-supporting $T$ cells and endogenous murine myeloid cells in lymphoid tissues, we used a CLL PDX model that allows CLL B- and T-cell engraftment and growth in recipient alymphoid mice ${ }^{26-28}$. Briefly, CLL patient PBMCs and patientderived T cells, previously activated in vitro, were injected retro-orbitally (ro) into NSG mice and allowed to engraft for 2 weeks prior to administering 3 weeks of duvelisib treatment (70 or $100 \mathrm{mg} / \mathrm{kg}$ via oral gavage once daily) (Fig. 4A). At a dose of $100 \mathrm{mg} / \mathrm{kg}$, duvelisib significantly reduced splenic CLL B-cell numbers relative to vehicle control (Fig. $4 \mathrm{~B}$ ), and significantly diminished the percentage of proliferating $\left(\mathrm{Ki}^{+} \mathrm{7}^{+}\right.$) splenic CLL B cells in a dose-dependent manner (Fig. 4C). The efficacy of duvelisib in tissueresident CLL cells is similar between unmutated (U-CLL) and mutated (M-CLL) IGHV CLL patients that are known to have distinct disease prognosis ${ }^{34,35}$ (Fig. 4D). Importantly, in all the patients, duvelisib significantly reduced the number of splenic patient-derived T cells (Fig. 4E) as well as decreasing the number of endogenous murine M2 macrophages (Fig. 4F).

Next, we examined the distinct functions of PI3K- $\delta$ and PI3K-y in CLL-cell engraftment and growth using the PDX model, but this time treating engrafted CLL cells and activated autologous T cells with PI3K-үi-

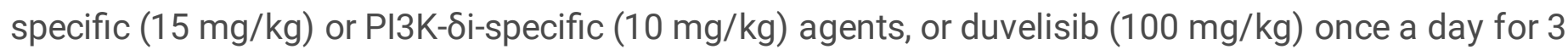
weeks. Both PI3K- $\delta i-$ and duvelisib treatments significantly decreased the number of leukemic B cells from both U-CLL and M-CLL patients in murine spleens (Fig. 5A and Fig. S3).

We next analyzed the growth of non-malignant cells in spleens from CLL-bearing mice treated as illustrated in Fig. 4A. Three-week treatment with PI3K-үi (15 mg/kg), PI3K-סi (10 mg/kg) or duvelisib (100 $\mathrm{mg} / \mathrm{kg}$ ) substantially decreased numbers of spleen-residing CLL-derived T-cell in mice treated with PI3K-үi or duvelisib, but not PI3K-סi (Fig. 5B). This was the case for all samples, regardless of IGHV mutation status (Fig. S3).

We then examined the distinct functions of PI3K- $\delta$ and PI3K- $y$ on murine splenic macrophage levels in the PDX model. Consistent with in vitro results, duvelisib and PI3K-үi monotherapy significantly reduced the 
number of murine M2 macrophages in the spleens after 3 weeks of treatment, whereas the PI3K- $\delta$ i did not induce a significant change (Fig. 5C).

Because of the difference in $\mathrm{IC}_{50}$ for duvelisib versus the isoform-specific agents (Table S1), we also used the same patient's cells to test the combination of the PI3K-үi- and PI3K-סi-specific agents. This indicated that in vivo treatment with the combination of PI3K- $\delta$ and PI3K-y inhibitors led to a significant decrease in splenic CLL B cells compared with the PI3K- $\delta$ i agent alone (Fig. 5D), supporting the advantage of dual $\mathrm{PI} 3 \mathrm{~K}-\delta, \gamma$ inhibition compared with PI3K-סi treatment alone.

Case study of a CLL patient salvaged with duvelisib after progression on ibrutinib. Intolerance and resistance to ibrutinib are emerging therapeutic problems, with the former developing in $50 \sim 63 \%$ of patients ${ }^{36}$ and the latter in $13 \sim 30 \%{ }^{37-39}$, often by selection for intraclonal variants harboring $B T K$ (C481S) or PLCG2 mutations ${ }^{22}$.

In this context, we report a patient (CLL 1570), who had failed treatment with fludarabine, FCR (fludarabine, cyclophosphamide, rituximab), tonsillar radiation, and BR (Bendamustine and Rituximab), before achieving a partial response to ibrutinib. Six years later, the patient became refractory to ibrutinib, and near the time of ibrutinib relapse, the leukemic clone contained BTKC481S and PLCG2 L845F mutations, along with mutations in BRCA2 (truncation in intron 26); MAP2K1 (MEK1; Q56P, subclonal); SF3B1 (K700E); TP53 (F113C and L330R, splice site 559 + 1G > A). Immediately after discontinuing ibrutinib (D0, Fig. 6), the patient was started on duvelisib (25 mg orally, twice daily) and continued for > 266 days, with only 2 short ( 13 and 3 days duration) treatment holds; the first, at day 50 , due to grade 2 elevations of ALT/AST and low level cytomegalovirus reactivation, and the second, at day 179, because of a temporary elevation of serum creatinine.

Duvelisib therapy led to a favorable response, designated as a partial response according to the International Workshop on Chronic Lymphocytic Leukemia (iwCLL) criteria ${ }^{40}$, at day 27 on duvelisib treatment. While thrombocytopenia and elevated absolute lymphocyte counts had developed on ibrutinib, these measures normalized following duvelisib treatment, with absolute values of lymphocytes decreasing by $\geq 50 \%$ on days $62-76,168-236,266$ and beyond (Fig. 6A). Since the patient rapidly developed a lymphocytosis $\left(>5 \times 10^{9}\right.$ cells/L), the fall in CLL cells presumably occurred by leukemic $B$ cells exiting tissue niches, including the BM which was the site of the most dramatic changes upon disease relapse. Specifically, the CLL cells in the BM fell from $60 \%$ before initiating duvelisib to $10 \%$ after 7 months on treatment (Fig. 6B). Additionally, other immune populations involved in leukemic B-cell control were either reduced (M2-like macrophages), increased (M1-like macrophages), or unchanged ( $T$ cells) compared with baseline values, for $>150$ days of duvelisib treatment (Fig. 6B).

CLL B cells from patients relapsing with ibrutinib are efficiently eliminated by duvelisib in a PDX model. Next, we attempted to model in vivo, using the PDX approach, the beneficial effects of duvelisib in this patient and in two others who had also developed ibrutinib-resistant disease. Like CLL1570 above, 
leukemic B cells from patient CLL1273 had at least a C481S BTK mutation; in contrast, neither BTK nor PLCG2 mutations were found in the leukemic cells from patient CLL1782.

NSG mice, engrafted with leukemic $B$ and T cells from each patient, were treated with ibrutinib $(25 \mathrm{mg} / \mathrm{kg})$ or duvelisib $(100 \mathrm{mg} / \mathrm{kg})$ daily for 3 weeks. As expected, based on the patients' clinical histories, in vivo ibrutinib treatment did not significantly affect CLL B-cell numbers in the spleens of recipient mice (Fig. 7A, 7B). However, after treating mice engrafted with each ibrutinib-resistant patient with duvelisib, splenic CLL B-cell numbers were significantly reduced regardless of BTK mutation status. Additionally, duvelisib, but not ibrutinib, significantly reduced the numbers of Ki $67^{+}$CLL B cells (Fig. 7C) and murine M2 macrophages (Fig. 7D) in the spleens. Interestingly, in these animals, no difference in the number of total T, Th1, or Th2 cells was observed with either duvelisib or ibrutinib treatment (Fig. S3). Collectively, findings in Figs. 6 and 7 indicate that the duvelisib responsiveness in ibrutinib-resistant patients, without or with BTKi resistance, is recapitulated in these PDX models. To our knowledge this is the first in vivo demonstration of ibrutinib-refractory CLL that are treated with duvelisib in clinical practice and PDX models, where effective suppression of BTK-mutated leukemic clones is achieved.

\section{Discussion}

BTK inhibitors, the current mainstays of treatment for patients with CLL, are administered continuously until disease progression or intolerance occurs. Despite excellent responses and progression-free survival, relapses occur and tumor cell acquired mutations ${ }^{41}$. Clinical outcomes after ibrutinib relapse are poor, and such patients often progress rapidly $21,22,42$. Since signals from $T$ cells and myeloid cells support the survival and growth of leukemic cells in tissue niches and can also thereby allow and advance disease progression and therapeutic refractoriness ${ }^{43,44}$, there is an ongoing need for therapies that circumvent BTKi drug resistance by directly targeting leukemic B cells directly and tumor-supportive cells, the latter thereby forestalling indirectly impeding leukemia-promoting pathways in the TME.

In this study, we dissected, in vitro and in vivo using a PDX model, the functions of dual inhibition of PI3K$\delta$ and PI3K- $y$ by duvelisib in primary CLL B, T and myeloid cells with the aim of determining if blocking another BCR-mediated pathway in CLL B cells and other functionally-relevant pathways in T cell and monocytes/macrophages by dual PI3K inhibition would avert BTK resistance. Our findings support previous observations of duvelisib's effects in B-cell malignancies $17,25,45$ and provide novel understandings of the drug's mechanisms of action by characterizing the individual contributions of PI3K- $\delta i$ and PI3K-үi, integral functions of duvelisib, in various cell types.

The four class I isoforms of PI3K - $a, \beta, y$ and $\delta$ - have overlapping, yet unique patterns of expression and biological functions. In contrast to the ubiquitously expressed PI3K- $\alpha$ and PI3K- $\beta$, the PI3K- $\gamma$ and $\mathrm{PI} 3 \mathrm{~K}-\delta$ isoforms are restricted to hematopoietic cells, with PI3K- $\delta$ contributing $~ 50 \%$ of the total PI3K activity in B lymphocytes and PI3K-y being more functionally restricted to T and myeloid cells ${ }^{46}$. Here we show that duvelisib targets both malignant B cells and the TME, consistent with achieving clinical improvement and impinging on trophic changes in the TME of R/R CLL patients ${ }^{47,48}$. 
For CLL B cells, we found that duvelisib $(0.1-1 \mu \mathrm{M})$ inhibits PI3K- $\delta$ signaling, thereby reducing leukemic B-cell proliferation and survival in vitro. Also, in vivo using a PDX model, duvelisib inhibited the migration of CLL B cells to the spleen through PI3K-סi, resulting in decreased leukemic B-cell numbers and indicating that preventing CLL cells from entering survival niches has a beneficial therapeutic effect. Moreover, since PI3K- $\delta$ modulates the IL-4R-induced phosphorylation of stromal cells which normally promotes B-cell adhesion and growth ${ }^{49,50}$, this TME effect likely affected the survival of those leukemic $B$ cells that did reach the spleen and other solid lymphoid tissues.

Both PI3K- $\delta$ i and PI3K-үi modulate cytokine synthesis in activated T cells ${ }^{46,51}$, and hence dual inhibition by duvelisib decreases production of IL-2, IFNy, and TNFa ${ }^{25}$. However, in vitro, we did not find a reduction in activated T-cell numbers with PI3K-סi nor PI3K-үi after stimulation with anti-CD3/28 beads and IL-2, whereas in vivo we did observe that pre-treatment with duvelisib and PI3K-үi blocked migration of T cells. These findings are compatible with PI3K-үi having minimal direct impact on CLL B-cell proliferative capacity, but ultimately leading to a reduction in T-cell numbers and amplification by reducing the ability of these cells to reach the spleen where growth can occur. Thus, like the direct trafficking inhibition of CLL B cells by PI3K- $\delta$ i seen above, direct trafficking inhibition of T cells by PI3K-үi leads to a fall in T cells. Since the majority of T cells that expand and support CLL B-cell growth in this PDX model are $\mathrm{CD} 4^{+}$their diminution indirectly leads to a reduction in CLL B cells by eliminating of their trophic effects. This was especially well exhibited in our PDX model that requires the support of T cells for CLL cell growth in tissue niches $^{52}$. Thus, the enhanced treatment efficacy provided by PI3K-үi might be due to an effect on the TME, such as on autologous T cells which are essential for CLL-cell survival in patients ${ }^{53}$ and in our PDX model $^{52}$. Although PI3K-סi has weak effects on T cells, PI3K-үi had a significantly greater effect on T-cell migration, and subsequent engraftment and growth in tissue niches. Dual inhibition of PI3K- $\delta, Y$ by duvelisib mirrored these effects on T-cell functions.

Finally, macrophages, which can dampen immune responses by producing cytokines that suppress cytotoxic T cell activation and function, were altered through PI3K-үi, and not PI3K- $\delta$. For example, the PI3K-y inhibitor, IPI-549, reduced the immune suppressive function of M2 macrophages ${ }^{15,16}$, whereas the $\mathrm{PI} 3 \mathrm{~K}-\delta i$ idelalisib has minimal impact on macrophage functions in vitro ${ }^{54}$. Consistent with this, when we assessed the role of PI3K- $\delta$ and PI3K-y in the endogenous macrophages of NSG mice in vitro and in vivo, duvelisib decreased M2 murine macrophage polarization and the support that such macrophages provided for CLL survival. These findings are consistent with previous findings in CLL $^{33}$ and T-cell lymphoma ${ }^{55}$. In addition, since T-cell subset distribution was changed in the TCL-1 murine model of $\mathrm{CLL}^{33}$ after macrophage-targeted treatment, the decreased T-cell growth that we observed in the PDX experiments might be contributed by the impaired macrophage subsets post duvelisib and PI3K-yi treatments. We similarly found that M2 macrophages were reduced in patient the CLL1570 upon initiation of duvelisib treatment for ibrutinib resistance, consistent with loss of support for CLL cell survival and promotion of an immune-stimulatory environment that activates effector $\mathrm{T}$ cells ${ }^{56}$. Thus, the 
reduced numbers of CLL B cells after duvelisib treatment could result directly by PI3K- $\delta i$ and indirectly by altering the supporting effects of T cells and macrophages via PI3K-үi.

Our PDX findings, demonstrating that duvelisib inhibited and ibrutinib did not the growth of CLL B cells from patients whose disease had progressed during ibrutinib therapy, regardless of the detection of BTK and/or PLCG2 mutations, could have substantial clinical relevance. Notably, patient CLL1570, who also previously progressed on ibrutinib, achieved a partial clinical response on duvelisib. Conspicuously, since, in this patient, the majority of ibrutinib-resistant cells resided in the BM, the duvelisib-induced redistribution lymphocytosis likely affected these cells, potentially by inhibiting the adhesion of CLL cells to $\mathrm{BM}$ mesenchymal stromal cells which requires the action of PI3K- $\delta^{49,50}$. Finally, the patient's response to duvelisib was manifested by an altered myeloid-cell phenotype that could abrogate support for CLL cell growth.

Regarding T cells, our PDX findings indicated that the reduction of T cells after duvelisib treatment could be due to the loss of B-CLL cells, impaired T-cell migration, and/or altered macrophage distributions. Differently, T-cell inhibition was not observed in mice receiving samples from patients with ibrutinibresistant disease, or clinically for patient CLL1570, who had progressed on ibrutinib. Since ibrutinib can affect $T$ cell function ${ }^{57-60}$, the extent to which the drug influences the T-cell population upon duvelisib treatment needs further evaluation, especially if the effects of duvelisib on T-cell activation are context dependent. Relevant are the effects of PI3K-үi, by genetic deletion or selective pharmacologic inhibitors like IPI-549, on the tumor microenvironment in solid tumors. Consistent with the effects observed in the CLL xenograft model, preclinical solid cancer models show that PI3K-үi results in a shift from pro-tumor M2-like macrophages to anti-tumor M1-like macrophages ${ }^{15,16}$. However, in contrast to the effects on T-cell migration observed in this CLL xenograft model, loss of PI3K-y in solid tumor models leads to enhanced T-cell recruitment and expansion, particularly within the $C D 8^{+}$cytotoxic subpopulation. The effects on $T$ cells in the latter context are thought to be mediated by reducing the immune suppressive function of myeloid cells, rather than a direct effect on T-cell proliferation or activation ${ }^{16}$. This underscores the importance of context and may reveal differences between the tumor microenvironment of hematologic malignancies and solid tumors.

Overall, our study provides the mechanistic rationale for using duvelisib in R/R CLL patients, although a larger number of patients will need to be studied to determine the scope of the clinical relevance of these initial observations. In this regard, although duvelisib was FDA-approved based on the phase 3 DUO trial ${ }^{18}$, patients who were previously exposed to ibrutinib or any other BTK inhibitors were excluded from that trial ${ }^{48}$, and hence data on post-BTK inhibitors are limited. Since duvelisib can cause serious toxicities (colitis, pneumonitis, transaminases, and severe infections) with the potential for rare fatal outcomes, further studies are warranted in patients refractory to BTKi and who are unable to receive or have failed BCL2 inhibition. To our knowledge, this is the first pre-clinical study supporting the use of duvelisib in patients who have progressed or are refractory to ibrutinib ${ }^{61}$, a current unmet need, especially if such patients are not candidates for BCL2-inhibitor therapy 
In summary, by studying primary CLL PBMCs in vitro and in vivo, we have delineated the mechanistic contributions of the individual and dual inhibitions of PI3K- $\delta$ and PI3K- $y$ in CLL. The multiple mechanistic inhibitory actions of duvelisib, which directly and indirectly affect leukemic and ancillary cells involved in the disease, support its use in R/R CLL.

\section{Materials And Methods}

CLL patient samples. Studies were approved by the Institutional Review Board of Northwell Health. Treatment-naïve and ibrutinib-resistant CLL subjects were selected using current iwCLL guidelines ${ }^{40}$. All patients provided written informed consent to participate. CLL clonal IGHV-IGHD-IGHJ sequences were determined by direct sequencing of PCR products from these samples as described ${ }^{62}$; cases with $^{2}$ germline identity $\geq 98 \%$ were considered U-CLL and those with germline identity $<98 \%$ were designated as M-CLL ${ }^{62}$. Targeted DNA sequencing for BTK C481S was performed in 2 CLL patients (CLL1782 and CLL1273) who exhibited clinical resistance to ibrutinib therapy. For a third ibrutinib-resistant patient (CLL1570), genomic profiling was conducted from a BM biopsy within 1 month of discontinuing ibrutinib.

Therapeutic response was assessed according to the iwCLL criteria ${ }^{40}$. Response assessments included evaluation of disease-related constitutional symptoms; focused physical examinations; complete blood counts and differential white blood cell counts; and bone marrow biopsy and computed tomography scans, when indicated.

CLL PDX model. Alymphoid NOD-scid IL-2Ry null (NSG) mice were housed 5 or less per cage, allowed free access to food and water, and maintained on a 12:12 hour light/dark schedule. All experiments were conducted in accordance with the Institutional Animal Care and Use Committee (IACUC) guidelines.

Engraftment and growth of human CLL cells in NSG mice was carried out as described ${ }^{26-28}$. Briefly, CD3 ${ }^{+}$ T cells were isolated from CLL PBMCs using EasySep (STEMCELL Technologies) and pre-activated with anti-CD3/CD28-coated Dynabeads (ThermoFisher Scientific) and IL-2 for 7 to 10 days. NSG mice were injected ro with $0.5 \times 10^{6}$ activated T cells and $20 \times 10^{6}$ autologous CLL PBMCs. Two weeks later, mice received duvelisib (70 or $100 \mathrm{mg} / \mathrm{kg}$ ), a selective PI3K-סi (IPI-3063; $10 \mathrm{mg} / \mathrm{kg}$ ), and/or a selective PI3K-үi (IPI-5243; $15 \mathrm{mg} / \mathrm{kg}$ ) 5 days per week for 3 weeks by oral gavage. $\mathrm{IC}_{50}$ inhibitory values of the different agents used in these assays for the four Class I PI3K isoforms are listed in Table S1.

A similar approach was used to evaluate CLL-cell growth in PDX model carrying ibrutinib-resistant CLL PBMCs. Leukemia-bearing mice were treated with either duvelisib $(100 \mathrm{mg} / \mathrm{kg} ; 5$ days a week via oral gavage) or ibrutinib (25 mg/kg; daily via drinking water). After 3 weeks of treatment, the spleens of mice were assessed by flow cytometry (Becton, Dickinson and Company) for patient-derived CLL B cells (gated on human $\mathrm{CD} 45^{+} \mathrm{CD} 19^{+} \mathrm{CD} 5^{+}$cells), proliferating CLL B cells (human $\mathrm{CD} 45^{+} \mathrm{CD} 19^{+} \mathrm{Ki}-67^{+}$cells), patientderived T cells (human $\mathrm{CD} 45^{+} \mathrm{CD} 3^{+} \mathrm{CD} 19^{-}$cells), or $\mathrm{M} 2$ macrophages (mouse $\mathrm{CD} 11 \mathrm{~b}^{+} \mathrm{F} 4 / 80^{+} \mathrm{mCD} 206^{+}$ cells or human $\mathrm{CD}_{206^{+}}$cells). 
In vitro CLL viability assessment. For viability assays, patient-derived CLL cells were incubated with PI3Ki (IPI-549) for 72 hours, then stained with the SYTOX Dead Cell Stains (Thermofisher) and analyzed by flow cytometry and FlowJo software (FlowJo, LLC). In the bone marrow-derived monocyte (BMDM)supported CLL-cell survival assay, CLL cells were added to the BMDMs at a ratio of 4:1 (monocytes to CLL cells); cultured 12 hours with M-CSF and IL-4, and then CLL-cell viability assessed via Guava ViaCount assay (MilliporeSigma) on days 0 and 9 of culture.

In vitro CLL B-cell proliferation assay. Human PBMCs from CLL donors obtained from whole blood from a commercial source (Bioreclamation IVT or ALLCELLS) were resuspended ( $1 \times 10^{6}$ cells $\left./ \mathrm{mL}\right)$ in RPMI-1640 medium containing $50 \mu \mathrm{M} \beta$-mercaptoethanol, 10\% heat-inactivated fetal bovine serum, $1000 \mathrm{units} / \mathrm{mL}$ penicillin and streptomycin. Cells were seeded at a density of $1 \times 10^{6}$ cells $/ \mathrm{mL} /$ well in a 24-well tissue culture plate. The PI3Ki IPI-549 was added at varying concentrations together with $1 \mu \mathrm{g} / \mathrm{mL} s C D 40 \mathrm{~L}$ (Gibco), $10 \mathrm{ng} / \mathrm{mL} \mathrm{IL}-10$ (Gibco) and $10 \mathrm{ng} / \mathrm{mL} \mathrm{IL}-2$ (PeproTech). Cells were incubated at $37^{\circ} \mathrm{C}$ for 5 days. Percent inhibition of $\mathrm{Ki}-67^{+} \mathrm{pAKT}{ }^{+} \mathrm{CLL}$ cells was calculated by flow cytometry, and these values were used to generate $\mathrm{EC}_{50}$ values using GraphPad Prism Software.

In vitro T-cell migration assay. CLL PBMCs were pre-treated with DMSO, duvelisib, PI3K-yi (IPI-549), or PI3K-סi (IPI-3063) for 24 hours and then allowed to migrate across a $6.5-\mathrm{mm}$ polycarbonate insert with a $5-\mu \mathrm{M}$ pore size in a Transwell (CoStar/Corning) plate in response to a 3-hour exposure at 37囚C to CXCL12 (300 ng/ml; R \& D systems). Cells were harvested from the lower chamber, and $\mathrm{CD} 3^{+} \mathrm{CD} 4^{+} \mathrm{CD} 8^{+}$cells quantified by flow cytometry. The percent inhibition of T-cell migration was calculated in response to each of the $3 \mathrm{PI} 3 \mathrm{Ki}$ used and compared to the DMSO control. $\mathrm{EC}_{50}$ values were then calculated using GraphPad Prism Software.

In vivo CLL B- and T-cell migration assay in NSG mice. CLL PBMCs pre-treated with PI3Kis for 48 hours were injected into NSG recipients. Twenty-four hours after injection, mice were sacrificed, and the number of CLL B cells in spleen and blood were measured. The migration of T cells from CLL patients was performed by intravenously injecting $5 \times 10^{6} \mathrm{~T}$ cells pre-activated for 7 days by anti-CD3/28 Dynabeads + IL-2 in the presence of PI3Ki.

M2 macrophage polarization. Bone marrow cells collected from 4-week-old wild-type male C57BL/6 mice $(\mathrm{N}=9)$ were plated in macrophage differentiation culture media (DMEM + 20\% FBS + 1\% Pen/Strep + $50 \mathrm{ng} / \mathrm{mL}$ M-CSF) for 6 days ${ }^{30}$. These bone marrow cells were then transferred to 96 -well plates and pretreated for 1 hour with various doses of duvelisib, PI3K-סi (IPI-3063), or PI3K-үi (IPI-549) in triplicates. After 1 hour, M-CSF $(50 \mathrm{ng} / \mathrm{mL})$ and IL-4 $(20 \mathrm{ng} / \mathrm{mL})$ were added to the culture. 48-hours later, RNA was extracted from cells per manufacturer's protocol (Qiagen) and Arg1 mRNA levels were determined by quantitative RT-PCR in triplicates. The threshold cycle (CT) values for Arg1 and Actb were obtained using an ABI7300 TaqMan instrument. IC50 calculations were obtained using Graphpad Prism by nonlinear regression of log inhibitor concentration versus percent inhibition of Arg1 expression. 
Intracellular staining for cytokine-producing cells. Samples were stimulated with PMA $(10 \mathrm{ng} / \mathrm{ml})$ and ionomycin $(1 \mu \mathrm{M})$ for 4 hours at $37^{\circ} \mathrm{C}$ in the presence of monensin $(10 \mu \mathrm{g} / \mathrm{ml})$, stained intracellularly for cytokine-producing live cells, and then analyzed by flow cytometry.

Statistical analyses. All statistical tests were performed using Prism v8 (GraphPad Software, Inc). Normality was assessed using the D'Agostino-Pearson Omnibus Test, and appropriate parametric and non-parametric analyses performed thereafter. Mann-Whitney $U$ tests were used to compare the numbers of CLL B and T cells and murine macrophages and the percentage of $\mathrm{Ki} 67^{+}$cells. An unpaired parametric $t$ test was used for the comparisons of CLL B-cell viability at day 5 . Figures shown are mean or mean \pm S.E.M, $P<.05$ was considered significant for all tests.

\section{Declarations}

\section{One Sentence Summary:}

Duvelisib directly inhibits CLL B cells, reduces support from T cells and macrophages, and is effective against CLL cells from patients whose disease has progressed during ibrutinib therapy, regardless of $B T K$ mutation status.

\section{References}

1. Kipps, T.J. et al. Chronic lymphocytic leukaemia. Nat Rev Dis Primers 3, 16096 (2017).

2. Fabbri, G. \& Dalla-Favera, R. The molecular pathogenesis of chronic lymphocytic leukaemia. Nat Rev Cancer 16 (2016).

3. Chiorazzi, N., Chen, S.S. \& Rai, K.R. Chronic Lymphocytic Leukemia. Cold Spring Harb Perspect Med (2020).

4. Burger, J.A. \& Gribben, J.G. The microenvironment in chronic lymphocytic leukemia (CLL) and other B cell malignancies: Insight into disease biology and new targeted therapies. Seminars in Cancer Biology 24, 71-81 (2014).

5. Ghia, P., Chiorazzi, N. \& Stamatopoulos, K. Microenvironmental influences in chronic lymphocytic leukaemia: the role of antigen stimulation. Journal of internal medicine 264, 549-562 (2008).

6. Munk Pedersen, I. \& Reed, J. Microenvironmental interactions and survival of CLL B-cells. Leuk Lymphoma 45, 2365-2372 (2004).

7. Burger, J.A. Chemokines and chemokine receptors in chronic lymphocytic leukemia (CLL): From understanding the basics towards therapeutic targeting. Semin Cancer Biol. 
8. Agarwal, A. et al. Genetic and cytokine changes associated with symptomatic stages of CLL. Leuk Res (2014).

9. Patel, K. \& Pagel, J.M. Exploring a Future for PI3K Inhibitors in Chronic Lymphocytic Leukemia. Curr Hematol Malig Rep (2019).

10. Frustaci, A.M. et al. Duvelisib for the treatment of chronic lymphocytic leukemia. Expert opinion on pharmacotherapy 21, 1299-1309 (2020).

11. Lampson, B.L. \& Brown, J.R. PI3Kdelta-selective and PI3Kalpha/delta-combinatorial inhibitors in clinical development for B-cell non-Hodgkin lymphoma. Expert opinion on investigational drugs 26, 12671279 (2017).

12. Peluso, M. et al. Duvelisib (IPI-145) Inhibits Malignant B-Cell Proliferation and Disrupts Signaling from the Tumor Microenvironment through Mechanisms That Are Dependent on PI3K- $\delta$ and PI3K-y. Blood 124 , abstract 328 (2014).

13. Winkler, D.G. et al. PI3K-delta and PI3K-gamma inhibition by IPI-145 abrogates immune responses and suppresses activity in autoimmune and inflammatory disease models. Chemistry \& biology 20, 1364-1374 (2013).

14. Hoellenriegel, J. et al. The phosphoinositide 3'-kinase delta inhibitor, CAL-101, inhibits B-cell receptor signaling and chemokine networks in chronic lymphocytic leukemia. Blood 118, 3603-3612 (2011).

15. De Henau, O. et al. Overcoming resistance to checkpoint blockade therapy by targeting PI3Kgamma in myeloid cells. Nature 539, 443-447 (2016).

16. Kaneda, M.M. et al. PI3Kgamma is a molecular switch that controls immune suppression. Nature 539, 437-442 (2016).

17. Balakrishnan, K. et al. The phosphoinositide-3-kinase (PI3K)-delta and gamma inhibitor, IPI-145 (Duvelisib), overcomes signals from the PI3K/AKT/S6 pathway and promotes apoptosis in CLL. Leukemia 29, 1811-1822 (2015).

18. Copiktra (duvelisib) [package insert]. Verastem, Inc. Needham, MA. http://www.verastem.com/pdf/prescribing-information.pdf (2018).

19. Flinn, I.W. et al. Duvelisib, a novel oral dual inhibitor of PI3K-delta,gamma, is clinically active in advanced hematologic malignancies. Blood 131, 877-887 (2018).

20. Imbruvica (ibrutinib) [package insert]. Pharmacyclics, Inc. Sunnyvale, CA. . https://www.accessdata.fda.gov/drugsatfda_docs/label/2019/205552s205029,210563s205004lbl.pdf (2019). 
21. Gango, A. et al. Dissection of subclonal evolution by temporal mutation profiling in chronic lymphocytic leukemia patients treated with ibrutinib. Int. J. Cancer(2019).

22. Woyach, J.A. et al. Resistance mechanisms for the Bruton's tyrosine kinase inhibitor ibrutinib. N. Engl. J. Med. 370, 2286-2294 (2014).

23. Zhang, S.Q., Smith, S.M., Zhang, S.Y. \& Lynn Wang, Y. Mechanisms of ibrutinib resistance in chronic lymphocytic leukaemia and non-Hodgkin lymphoma. Br. J. Haematol. 170, 445-456 (2015).

24. Lampson, B.L.B., J. R. Are BTK and PLCG2 mutations necessary and sufficient for ibrutinib resistance in chronic lymphocytic leukemia. . Expert Review of Hematology 11, 185-194 (2018).

25. Dong, S. et al. IPI-145 antagonizes intrinsic and extrinsic survival signals in chronic lymphocytic leukemia cells. Blood 124, 3583-3586 (2014).

26. Chen, S.S. Method for generating a patient-derived xenograft model of CLL, in Chronic Lymphocytic Leukemia Methods and Protocols, Vol. Methods in Molecular Biology volume 1881. (ed. S.N. Malek) 165 (Humana Press, New York, NY; 2018).

27. Patten, P.E. et al. Chronic lymphocytic leukemia cells diversify and differentiate in vivo via a nonclassical Th1-dependent, Bcl-6-deficient process. JCl insight 1 (2016).

28. Chen, S.S. \& Chiorazzi, N. Murine genetically engineered and human xenograft models of chronic lymphocytic leukemia. Seminars in hematology 51, 188-205 (2014).

29. Arlauckas, S.P. et al. Arg1 expression defines immunosuppressive subsets of tumor-associated macrophages. Theranostics 8, 5842-5854 (2018).

30. Ying, W., Cheruku, P.S., Bazer, F.W., Safe, S.H. \& Zhou, B. Investigation of macrophage polarization using bone marrow derived macrophages. Journal of visualized experiments : JoVE (76). doi, $10.3791 / 50323$ (2013).

31. Ten Hacken, E. \& Burger, J.A. Microenvironment interactions and B-cell receptor signaling in Chronic Lymphocytic Leukemia: Implications for disease pathogenesis and treatment. Biochimica et biophysica acta 1863, 401-413 (2016).

32. van Attekum, M.H.A. et al. Macrophages confer survival signals via CCR1-dependent translational MCL-1 induction in chronic lymphocytic leukemia. Oncogene 36, 3651-3660 (2017).

33. Galletti, G. et al. Targeting Macrophages Sensitizes Chronic Lymphocytic Leukemia to Apoptosis and Inhibits Disease Progression. Cell reports 14, 1748-1760 (2016).

34. Damle, R.N. et al. B-CLL patients can be divided into two distinct clinical categories based on CD38 expression and Ig V gene mutation status. Blood 92, 431 a (1998). 
35. Hamblin, T.J., Davis, Z., Gardiner, A., Oscier, D.G. \& Stevenson, F.K. Unmutated Ig V(H) genes are associated with a more aggressive form of chronic lymphocytic leukemia. Blood 94 (1999).

36. Mato, A.R. et al. Real-world outcomes and management strategies for venetoclax-treated chronic lymphocytic leukemia patients in the United States. Haematologica 103, 1511-1517 (2018).

37. Byrd, J.C. et al. Targeting BTK with Ibrutinib in Relapsed Chronic Lymphocytic Leukemia. New England Journal of Medicine 369, 32-42 (2013).

38. Furman, R.R. et al. Ibrutinib Resistance in Chronic Lymphocytic Leukemia. New England Journal of Medicine 370, 2352-2354 (2014).

39. Woyach, J.A. et al. Resistance Mechanisms for the Bruton's Tyrosine Kinase Inhibitor Ibrutinib. New England Journal of Medicine 370, 2286-2294 (2014).

40. Hallek, M. et al. Guidelines for the diagnosis and treatment of chronic lymphocytic leukemia: a report from the International Workshop on Chronic Lymphocytic Leukemia updating the National Cancer Institute-Working Group 1996 guidelines. Blood 111, 5446-5456 (2008).

41. Stephens, D.M. \& Byrd, J.C. How we manage ibrutinib intolerance and complications in patients with chronic lymphocytic leukemia. Blood, blood-2018-2011-846808 (2019).

42. Quinquenel, A. et al. Prevalence of BTK and PLCG2 mutations in a real-life CLL cohort still on ibrutinib after 3 years: a FILO group study. Blood 134, 641-644 (2019).

43. Fecteau, J.F. \& Kipps, T.J. Structure and function of the hematopoietic cancer niche: focus on chronic lymphocytic leukemia. Front Biosci (Schol Ed) 4, 61-73 (2012).

44. Zhang, S. \& Kipps, T.J. The pathogenesis of chronic lymphocytic leukemia. Annual review of pathology 9, 103-118 (2014).

45. Faia, K. et al. The phosphoinositide-3 kinase (PI3K)-delta,gamma inhibitor, duvelisib shows preclinical synergy with multiple targeted therapies in hematologic malignancies. PloS one 13, e0200725 (2018).

46. Sasaki, T. et al. Function of PI3Kgamma in thymocyte development, T cell activation, and neutrophil migration. Science 287, 1040-1046 (2000).

47. Rodrigues, D.A., Sagrillo, F.S. \& Fraga, C.A.M. Duvelisib: A 2018 Novel FDA-Approved Small Molecule Inhibiting Phosphoinositide 3-Kinases. Pharmaceuticals (Basel) 12 (2019).

48. Flinn, I.W. et al. The phase 3 DUO trial: duvelisib vs ofatumumab in relapsed and refractory CLL/SLL. Blood 132, 2446-2455 (2018). 
49. Ali, A.Y. et al. Expression and function of phosphoinositide 3-kinase delta in mesenchymal stromal cells from normal and leukaemic bone marrow. Br. J. Haematol. 185, 883-887 (2019).

50. Bilancio, A. et al. Key role of the p110delta isoform of PI3K in B-cell antigen and IL-4 receptor signaling: comparative analysis of genetic and pharmacologic interference with p110delta function in $B$ cells. Blood 107, 642-650 (2006).

51. Martinelli, S. et al. Idelalisib impairs T-cell-mediated immunity in chronic lymphocytic leukemia. Haematologica 103, e598-e601 (2018).

52. Bagnara, D. et al. Autologous human T cells and allogeneic antigen-presenting cells permit effective adoptive transfer of B-CLL cells into immune deficient mice. Blood in press (2009).

53. Roessner PM, S.M. T-cells in chronic lymphocytic leukemia: Guardians or drivers of disease? . Leukemia 34, 2012-2024 (2020).

54. Palazzo, A. et al. The PI3Kdelta-Selective Inhibitor Idelalisib Minimally Interferes with Immune Effector Function Mediated by Rituximab or Obinutuzumab and Significantly Augments B Cell Depletion In Vivo. J. Immunol. 200, 2304-2312 (2018).

55. Horwitz, S.M. et al. Activity of the PI3K-delta,gamma inhibitor duvelisib in a phase 1 trial and preclinical models of T-cell lymphoma. Blood 131, 888-898 (2018).

56. Sica, A. \& Mantovani, A. Macrophage plasticity and polarization: in vivo veritas. J Clin Invest 122, 787-795 (2012).

57. Long, M. et al. Ibrutinib treatment improves T cell number and function in CLL patients. J. Clin. Invest. 127, 3052-3064 (2017).

58. Parry, H.M. et al. Long-Term Ibrutinib Therapy Reverses CD8(+) T Cell Exhaustion in B Cell Chronic Lymphocytic Leukaemia. Front. Immunol. 10, 2832 (2019).

59. Solman, I.G.B., L. K.; Hoh, H. Y.; Kipps, T. J.; Burger, J. A.; Barrientos, J. C.; O'Brien, S.; Mulligan, S. P.; Kay, N. E.; Hillmen, P.; Byrd, J. C.; Lal, I. D.; Dean, J. P. ; Mongan, A. Ibrutinib restores immune cell numbers and function in first-line and relapsed/refractory chronic lymphocytic leukemia. . Leukemia Research, Epub (2020).

60. J. C. Barrientos, S.O.B., J. R. Brown, N. E. Kay, N. M. Reddy, S. Coutre, C. Tam, S. Mulligan, U. Jaeger, S. Devereux, C. Pocock, T. Robak, S. J. Schuster, A. Schuh, D. Gill, A. Bloor, C. Dearden, C. Moreno, G. Cull, M. Hamblin, J. A. Jones, K. Eckert, I. G. Solman, S. Suzuki, E. Hsu, D. F. James, J. C. Byrd, P. Hillmen. Improvement in Parameters of Hematologic and Immunologic Function and Patient Well-being in the Phase III RESONATE Study of Ibrutinib Versus Ofatumumab in Patients With Previously Treated Chronic Lymphocytic Leukemia/Small Lymphocytic Lymphoma. . Clinical Lymphoma, Myeloma \& Leukemia 18, 803-813 (2018). 
61. Porcu, P. et al. Clinical Activity of Duvelisib (IPI-145), a Phosphoinositide-3-Kinase- $\delta, Y$ Inhibitor, in Patients Previously Treated with Ibrutinib. Blood 124, Abstract 3335 (2014).

62. Fais, F. et al. Chronic lymphocytic leukemia B cells express restricted sets of mutated and unmutated antigen receptors. J Clin Invest 102, 1515-1525 (1998).

63. Evans, C.A. et al. Discovery of a Selective Phosphoinositide-3-Kinase (PI3K)-gamma Inhibitor (IPI549) as an Immuno-Oncology Clinical Candidate. ACS Med. Chem. Lett. 7, 862-867 (2016).

\section{Table 1}

Table 1

Mutation characterization of ibrutinib resistant CLL samples used in this study.

\begin{tabular}{|lllll|}
\hline Patient Identifier & IGHV & Ibrutinib Progression & BTKmutation & PLCG2 mutation \\
\hline CLL1782 & Unmutated & yes & wildtype & \\
\hline CLL1273 & Mutated & yes & C481S & \\
\hline CLL1570 & Mutated & yes & C481S & PLCG2 L845F \\
\hline
\end{tabular}

\section{Figures}
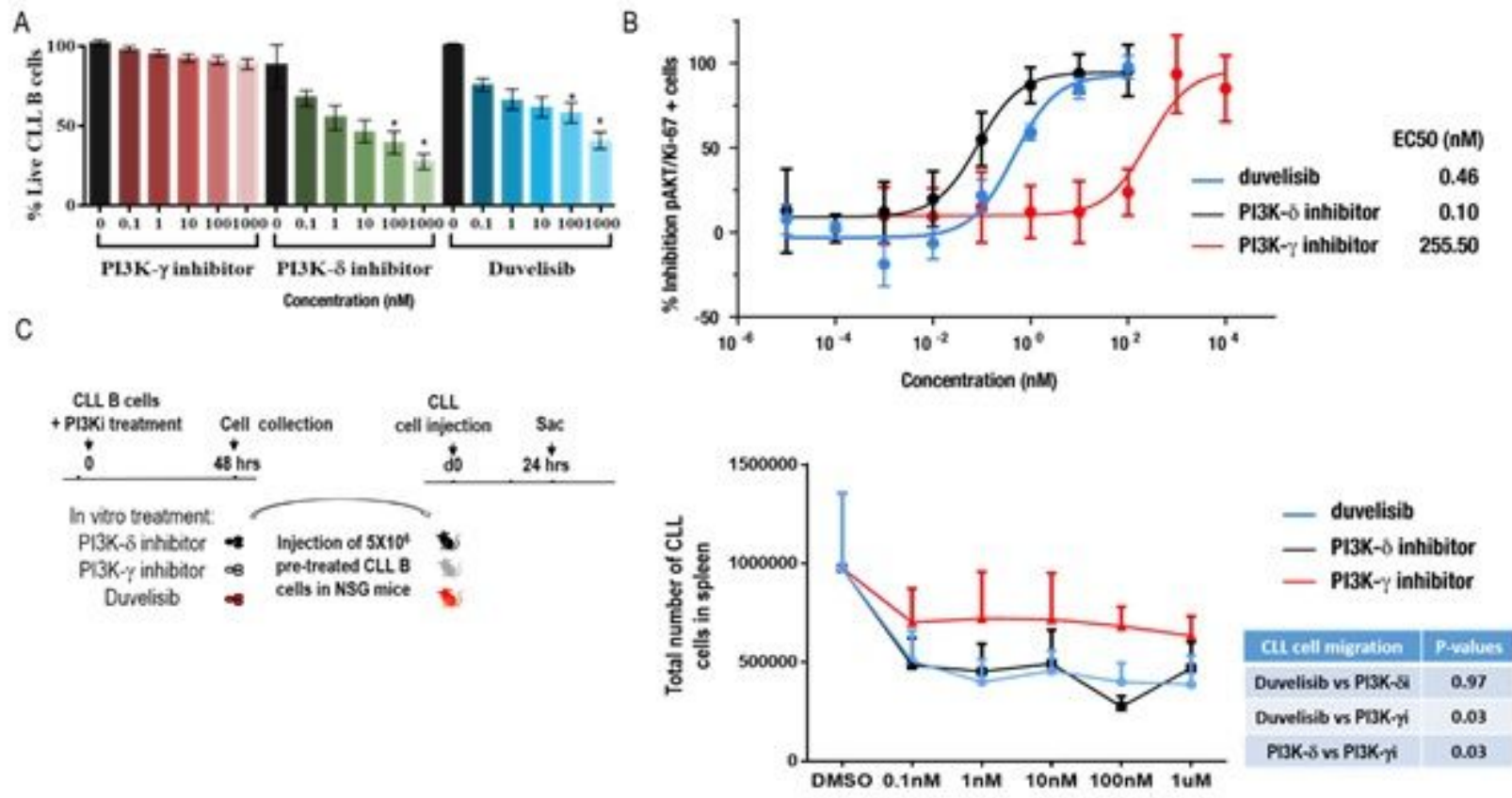

Figure 1 
Inhibition of PI3K-ס impairs CLL B-cell proliferation, survival, and migration in vitro and in vivo. Dosedependent effects of PI3K-di monotherapy, PI3K-gi monotherapy, and dual PI3K-d,gi inhibition with duvelisib analyzed in 4 CLL cases for (A) survival of CLL cells 96 hour post incubation, (B) CLL-cell proliferation after stimulation by soluble CD40L, IL-2, and IL-10 for 72 hours, and (C) in vivo migration after treating CLL B cells with PI3K-gi, PI3K-di or duvelisib at doses ranging from 0 to $1 \mathrm{uM}$ for 48 hours. $5 \times 106$ live cells obtained from each culture condition were then injected into NSG mice. Data shown are represented as mean $+\mathrm{S}$.E.M. * indicates $\mathrm{P}<.05$, ** $\mathrm{P} \leq .01$ analyzed by 2 -way ANOVA

A

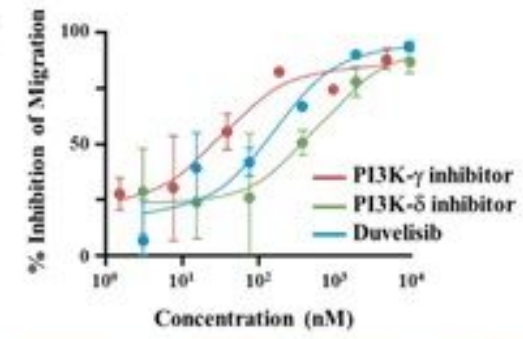

\begin{tabular}{|c|c|c|c|}
\hline $\begin{array}{l}\text { CXCL.12- } \\
\text { Induced } \\
\text { migration }\end{array}$ & $\begin{array}{l}\text { Duvelisib } \\
\mathrm{EC}_{\mathrm{M}}(\mathrm{n} \mathrm{M})\end{array}$ & $\begin{array}{c}\text { PISK -8 } \\
\text { inhibitor } \\
\mathrm{EC}_{\mathrm{S}}(\mathrm{aM})\end{array}$ & $\begin{array}{l}\text { PLBK-y } \\
\text { inhibitor } \\
\mathrm{KC}_{\mathrm{n}} \text { (8M) }\end{array}$ \\
\hline CDs: & $128=39$ & $630 \pm 71$ & $17 \pm 17$ \\
\hline $\mathrm{CD}^{*}$ & $164=80$ & $726 \pm 230$ & $20=21$ \\
\hline CDs: & $106 \pm 77$ & $423 \pm 290$ & $13 \neq 15$ \\
\hline
\end{tabular}

B
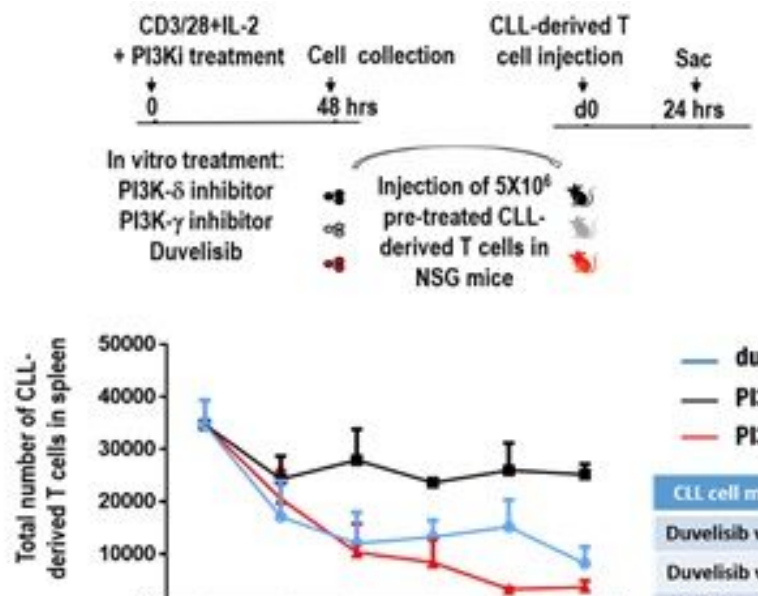

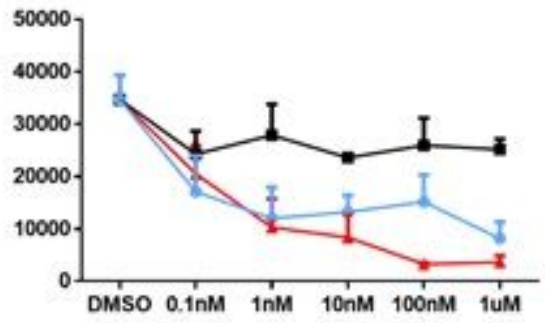

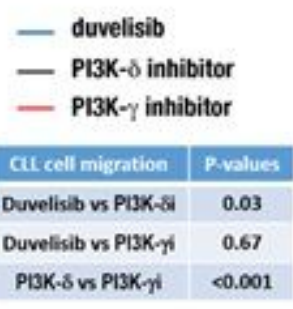

\section{Figure 2}

Inhibition of PI3K-y impairs migration of CLL-derived T cells. (A) CXCL12-induced migration of CD3+ T cells from 3 CLL patients. EC50 are represented as mean \pm S.D. (B) Schematic of in vivo migration model of CLL T cells. Equal numbers of T cells, collected after treatment with PI3K-gi, PI3K-dl, or duvelisib, were injected into NSG mice ro. T-cell migration to the spleen, and the contributions of the PI3K-gi vs PI3K-di components of duvelisib were evaluated 24 hour later. Total 4 CLL cases were tested. Absolute number of T cell migrated to spleen were calculated by flow cytometry and shown by mean \pm S.E.M. Statistical analysis was done by 2-way ANOVA. Duvelisib vs. PI3K-di: $\mathrm{P}=0.03$; PI3K-gi vs. PI3K-di: $\mathrm{P}<0.01$. 

A
Proliferation:
CLL cells added to coculture with M-CSF 50ng/mL Duvelisib
M2 macrophages (4:1)

Bone marrow isolated from 3to 4-week- old C57BL6 mcie

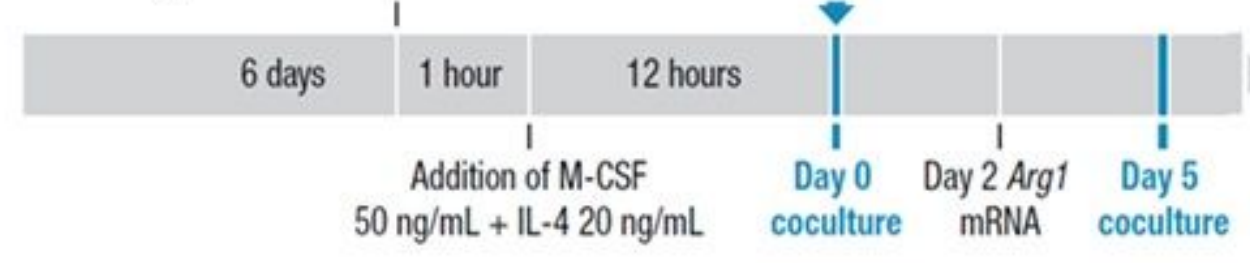

B

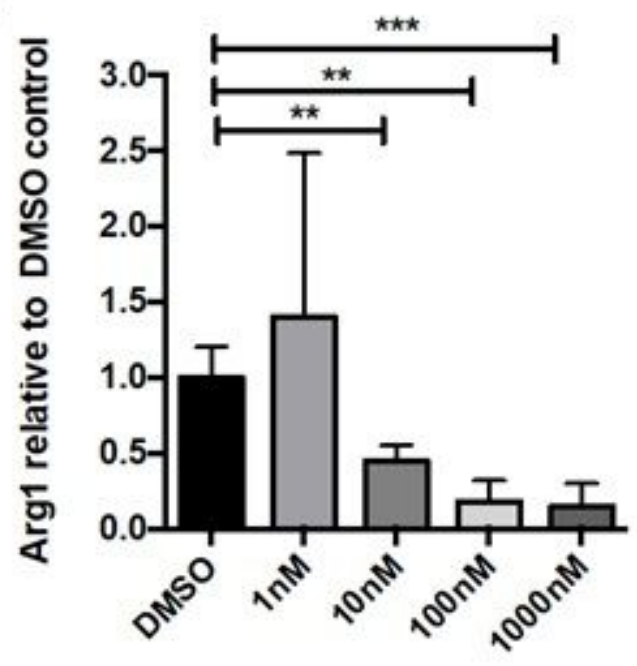

C

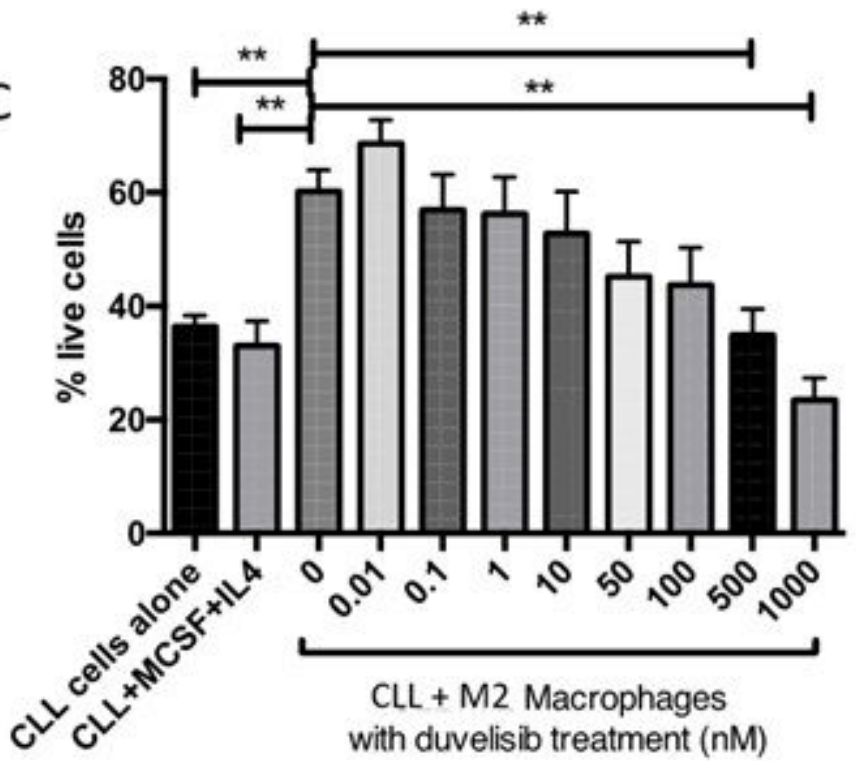

Figure 3

Inhibition of PI3K-y reduces M2 macrophage polarization and M2 macrophage support of CLL B-cell survival. (A) Schematic of in vitro CLL and M2 macrophage co-culture system and assays. (B) Arg1 expression levels determined by RT-qPCR in M2 macrophage-polarized BMDMs treated with duvelisib. (C) Effect of the addition of duvelisib to co-cultures of CLL B cells with M2-polarized macrophages. ${ }^{*}<0.05$, ** $P \leq 0.01,{ }^{* \star *} P \leq 0.001$. 

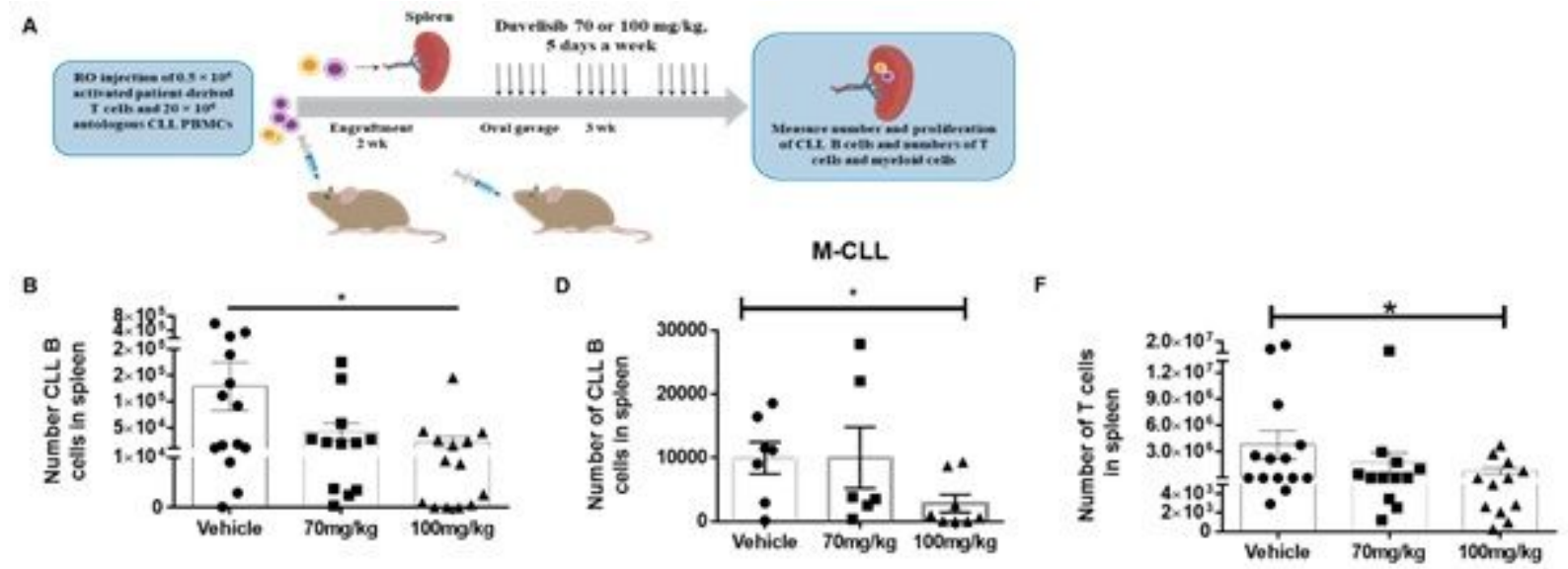

c
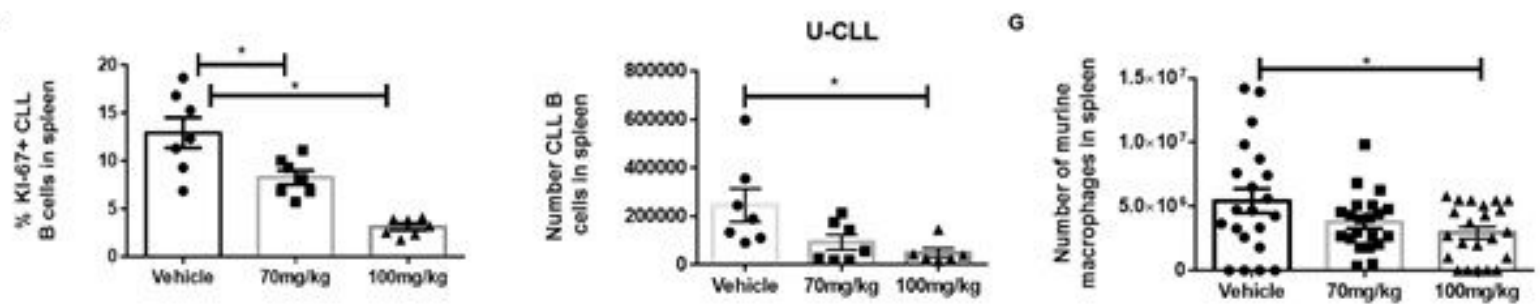

Figure 4

Duvelisib treatment alters microenvironment that supports CLL-cell growth. (A) Schematic of PDX model. (B-F) Quantification of human CLL B and T cells and murine myeloid cells by flow cytometry in mice receiving cells from $2 \mathrm{U}-\mathrm{CLL}$ and $2 \mathrm{M}-\mathrm{CLL}$ patients. 3-5 mice per patient per condition. (B) Number of CLL $B$ cells in the spleens after duvelisib treatment $(100 \mathrm{mg} / \mathrm{kg})$. (C) Number of CLL B cells in the cell cycle after duvelisib treatment (70 or $100 \mathrm{mg} / \mathrm{kg}$ ). (D) Number of CLL from M-CLL and U-CLL patients after duvelisib treatment (70 or $100 \mathrm{mg} / \mathrm{kg}$ ). (E) Number of T cells in mice treated with duvelisib $(100 \mathrm{mg} / \mathrm{kg})$. (F) Number of murine CD11b+GRlowLY-6Clow macrophages in recipient mouse spleens. * indicates $\mathrm{P}<$ $.05, * * \mathrm{P} \leq .01$. 
A

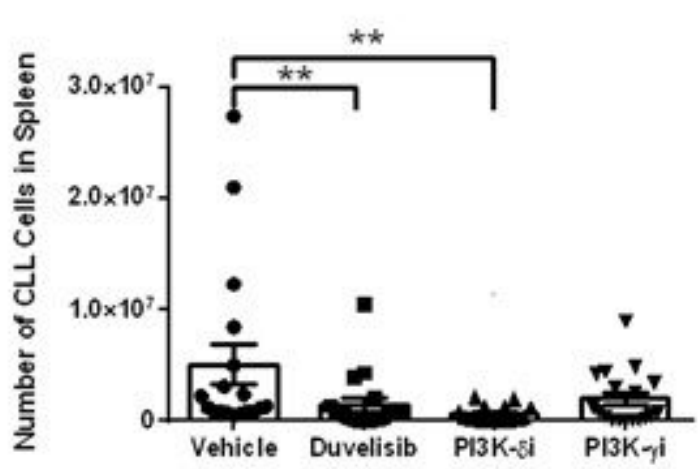

C

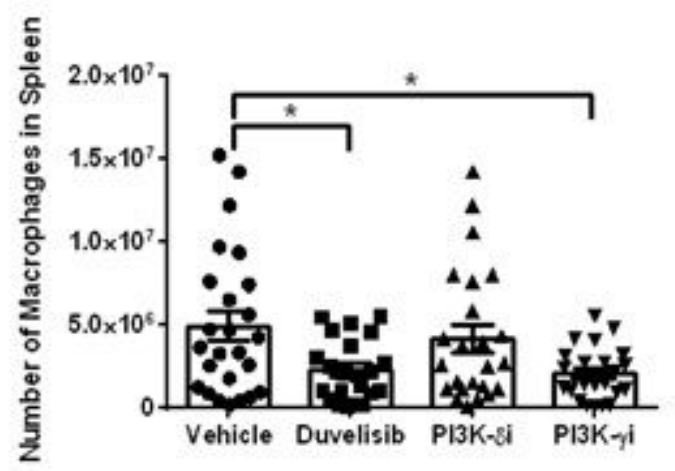

B

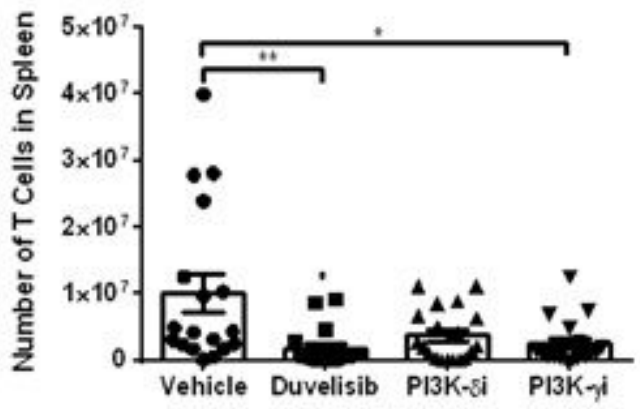

D

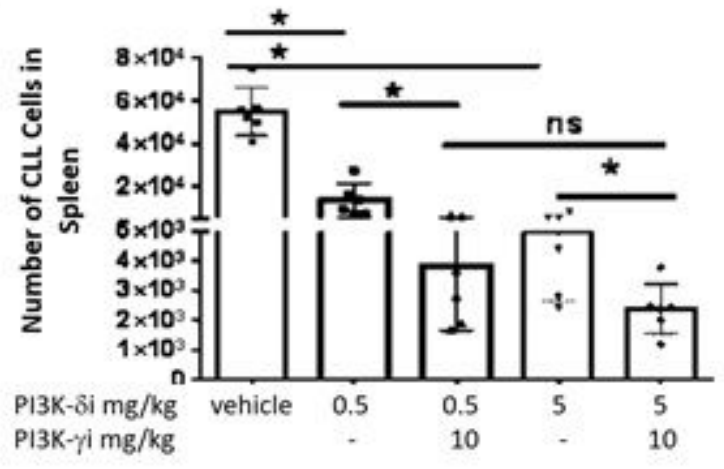

Figure 5

Inhibition of PI3K- $\delta$ and PI3K-y impairs CLL B-cell proliferation and alters T cells and myeloid cells in a xenograft model. Numbers of (A) CLL B cells, (B) CLL-derived T cells, and (C) macrophages in the spleens of mice treated 5 days a week for 3 weeks with PI3K-gi ( $15 \mathrm{mg} / \mathrm{kg})$, PI3K-di $(10 \mathrm{mg} / \mathrm{kg} ; \mathrm{IPI}-3063)$ or duvelisib (100 mg/kg). (D) Number of CLL B cells in the spleen after exposure to the combination of PI3K$\mathrm{d}, \mathrm{g}$ or PI3K-d alone. ${ }^{*} \mathrm{P}<0.05, * * \mathrm{P} \leq 0.01$.

A
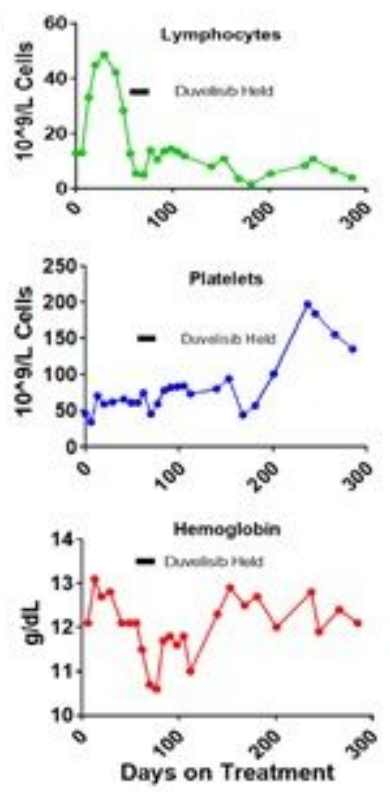

B
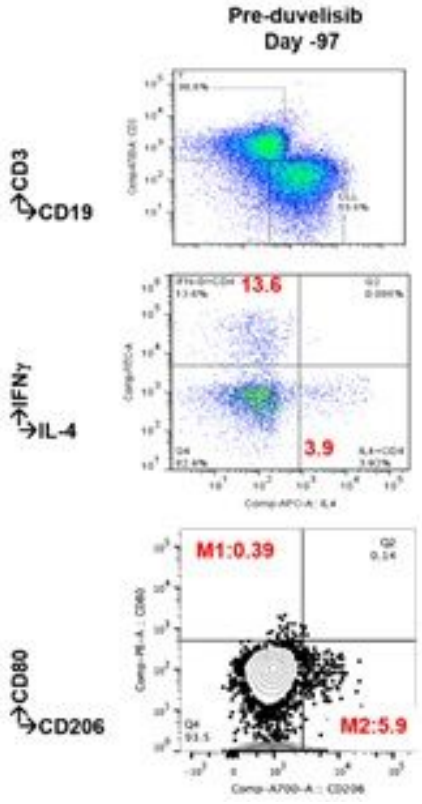
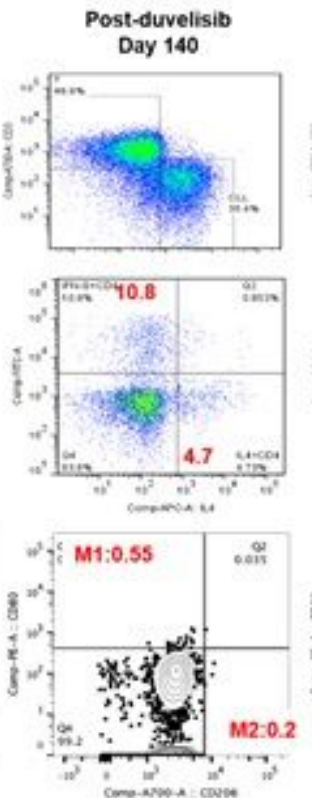
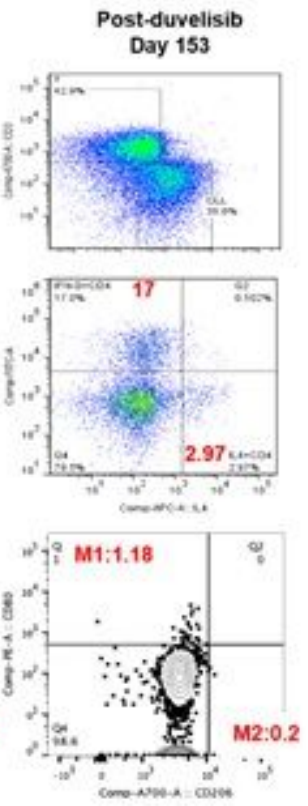

Figure 6 
Analyses of a CLL patient with BTK and other genomic mutations who failed ibrutinib and several other therapies but achieved a partial response on duvelisib. (A) Lymphocyte, platelet, and hemoglobin counts for patient CLL1570 after receiving duvelisib (Day 0). The bars indicate times when duvelisib was temporarily held. Patient received once-daily, oral ibrutinib $(420 \mathrm{mg})$ for 1,344 days. Upon relapse, treatment was changed to twice-daily oral duvelisib $(250 \mathrm{mg})$ for about 400 days. (B) Characterization of CLL CD19+ B cells, CD3+ T cells, and CD4+Th1 and CD4+Th2 cells and bone marrow M1- and M2macrophages by flow cytometry, before and over time after initiation of duvelisib.

A
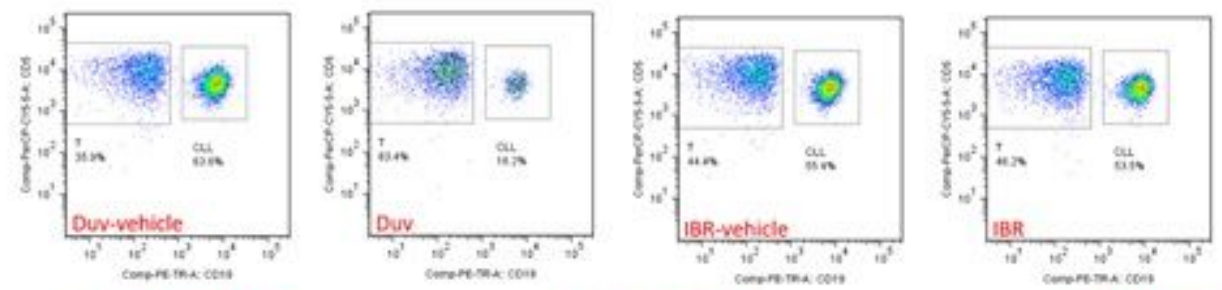

B Cu1782 wild-type BTK cu.1273 втK mut

CL1570 BTK mut
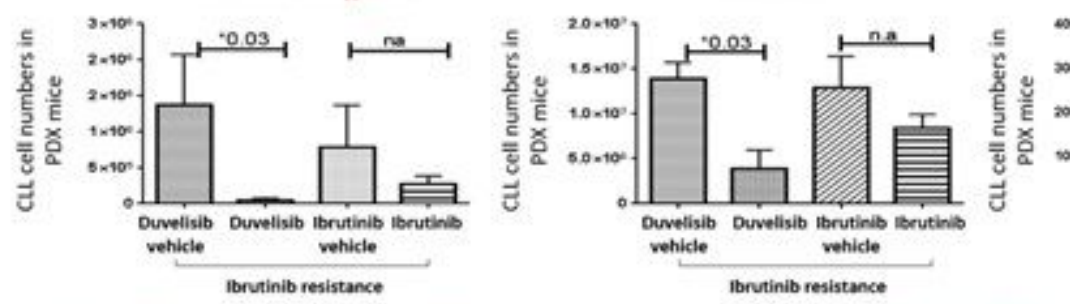

C Wild-type BTK

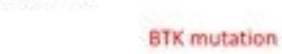

D
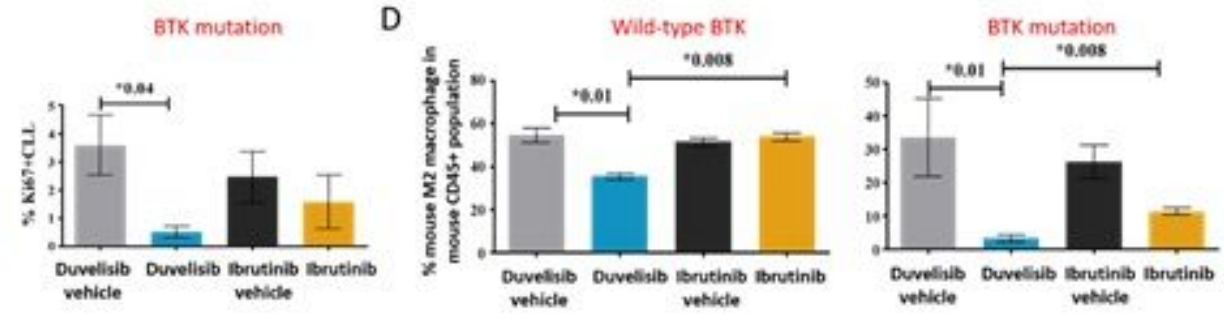

Figure 7

CLL B cells from patients unresponsive to ibrutinib are efficiently eliminated by duvelisib in a PDX model. (A) Representative flow cytometry tracings showing CLL B cells and T cells from mice that received treatment with duvelisib (Duv), duvelisib vehicle control (Duv-vehicle), ibrutinib (IBR), or ibrutinib vehicle control (IBR-vehicle). (B) Number of CLL cells in spleen obtained from mice engrafted with ibrutinibresistant samples carrying wild type (CLL1782) or at least a C481S mutation in BTK (CLL1273 and CLL1570). (C) Proliferation (Ki67+) of CLL B cells from patients with wildtype or mutant BTK. (D) Effects of duvelisib treatment on murine macrophage numbers in the spleens of mice engrafted with samples from ibrutinib-resistant CLL patients. * indicates $\mathrm{P}<.05$, ** $\mathrm{P} \leq .01$.

\section{Supplementary Files}

This is a list of supplementary files associated with this preprint. Click to download.

- SupplementalMaterial.pdf 